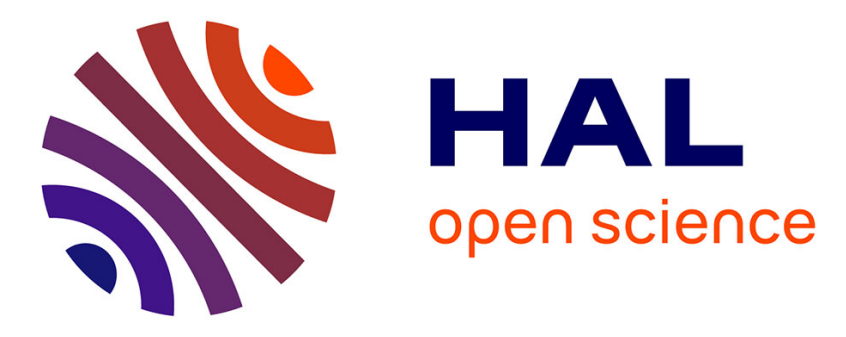

\title{
Controlled contractive sets for low-complexity constrained control
}

Sarmad Munir, Morten Hovd, Guillaume Sandou, Sorin Olaru

\section{To cite this version:}

Sarmad Munir, Morten Hovd, Guillaume Sandou, Sorin Olaru. Controlled contractive sets for lowcomplexity constrained control. IEEE Multi-Conference on Systems and Control (MSC 2016), Sep 2016, Buenos Aires, Argentina. 10.1109/cacsd.2016.7602546 . hal-01381706

\section{HAL Id: hal-01381706 \\ https://hal-centralesupelec.archives-ouvertes.fr/hal-01381706}

Submitted on 14 Oct 2016

HAL is a multi-disciplinary open access archive for the deposit and dissemination of scientific research documents, whether they are published or not. The documents may come from teaching and research institutions in France or abroad, or from public or private research centers.
L'archive ouverte pluridisciplinaire HAL, est destinée au dépôt et à la diffusion de documents scientifiques de niveau recherche, publiés ou non, émanant des établissements d'enseignement et de recherche français ou étrangers, des laboratoires publics ou privés. 


\title{
Controlled contractive sets for low-complexity constrained control
}

\author{
Sarmad Munir, ${ }^{1}$ Morten Hovd, ${ }^{1}$ Guillaume Sandou, ${ }^{2}$ and Sorin Olaru ${ }^{2}$
}

\begin{abstract}
Explicit constrained control is relatively simple when a controlled contractive set is available. However, the complexity of the explicit controller will depend on the complexity of the controlled contractive set. The ability to design a low complexity controlled contractive set is therefore desirable. Most methods for finding controlled contractive sets either assume the use of a constant linear state feedback, or is based on reachable set computations. In the first case, the assumption of a constant linear state feedback is restrictive (as MPC controllers for linear systems are typically piecewise affine), and in the second case the complexity of the controlled contractive set may be very high.

Initial results on the construction of low complexity controlled contractive sets without assuming linear state feedback were reported at the IFAC World Congress in 2014. The present paper addresses shortcomings in the previous results, including the ability to handle identical modes in series (corresponding to a non-diagonalizable $A$ matrix) and oscillatory modes. The paper suggests a method to find a flexible complexity controlled contractive set.
\end{abstract}

\section{INTRODUCTION}

Model Predictive Control (MPC) has been used in industry for decades. There is a wide application of MPC in industries nowadays [1]. From slow speed chemical processes to fast systems in automotive sector, MPC is highly useful [2]. MPC uses the system model information to obtain control law. It allows operating of a system for long periods without expert intervention [3]. Standard MPC is based on online solutions of optimization problems. Due to its computational complexity, its application is limited to the systems with slow dynamics which are not safety critical [4]. Explicit MPC provides an answer to these limitations of standard MPC, by formulating the MPC problem as multi-parametric problem. Instead of solving problem online, it can be solved offline and optimal control law can be given as piecewise affine (PWA) functions of the present state [5]. Therefore the online MPC computation is transformed to simple evaluation of a PWA function [6]. This allows implementation of simple hardware with fast sampling rate. However, as the problem size increases, number of the regions of the explicit solution and the memory required for storing the explicit solution increases rapidly. This limits the use of explicit MPC to a

\footnotetext{
*The work leading to these results has received funding from the People Programme (Marie Curie Actions) of the European Union's Seventh Framework Programme (FP7/2007-2013) under REA grant agreement no 607957 (TEMPO)

${ }^{1}$ The authors are with the Engineering Cybernetics Department, Norwegian University of Science and Technology, N-7491 Trondheim, Norway. sarmad.munireitk.ntnu.no, morten.hovdeitk.ntnu.no ${ }^{2}$ The authors are with the Automatic Control Department, Supelec, 3 rue Joliot Curie, 91192 France. guillaume.sandoudsupelec.fr, sorin.olarudcentralesupelec.fr
}

system with modest number of states and short prediction horizons. Thus, the complexity reduction in explicit Model Predictive Control becomes next big challenge.

There has been significant research going on to find approximate solutions in order to simplify explicit solutions. A simpler solution by directly approximating the control law is formulated in [7]. Vertex Control approaches can be utilized for simpler controller designs. In the vertex control approach, an admissible input corresponding to vertices is used for controller design [8]. A common way to do this would be to calculate the feasible region for an MPC formulation with guaranteed stability. However, the calculation of feasible regions for MPC may also be computationally complex [9].

A simpler approach is using explicit constrained control with the help of controlled contractive set. In this case, complexity of the Explicit MPC depends on the contractive set complexity. Therefore, finding a low complexity contractive set is essential for complexity reduction in Explicit MPC. The procedure in [10] converges to the maximal controlled contractive set with a specified contraction factor. However, the complexity of the resulting set may be very high. In [4], a non-iterative procedure for obtaining lowcomplexity contractive set is proposed and it is shown how the contractive set can be used for designing a controller for a system with state and input constraints. It also proposed an approach to handle oscillatory modes given sufficient contractive dynamics. However, the approach is not generally applicable to systems with oscillatory modes, and is not applicable to identical modes in series. Furthermore, the contractive set obtained in [4] is of fixed complexity, without any means of trading off complexity versus the size of the contractive set. The main purpose of this paper is to describe a flexible approach to obtain a low complexity contractive sets enabling the design of low complexity explicit constrained control, which also handles identical and oscillatory modes in series and allows a trade-off between the size of the controlled contractive set and the complexity of the set.

Section 2 describes how to find a contractive set using an optimization based approach. In section 3, different solutions are merged together in order to find a larger contractive set. In section 4, two methods are introduced to simplify complex contractive sets, first method introduces circumscribed ellipsoid technique to simplify the contractive set while second method directly removes hyperplanes in order to obtain a low complexity contractive set. In section 5, polytopic contractive sets are considered for controller design, as polytopic contractive sets are natural starting points for designing explicit constrained controllers. Section 6 provides examples of these approaches and conclusion is drawn in section 7 . 


\section{Optimization BASED CALCULATION FOR Controlled Contractive Sets}

Consider the discrete-time system

$$
x_{k+1}=A x_{k}+B u_{k}
$$

with state and input constraints given by $\mathcal{X}=\left\{x \mid H_{x} x \leq\right.$ $\left.h_{x}\right\}$ and $\mathcal{U}=\left\{u \mid H_{u} u \leq h_{u}\right\}$ respectively.

Definition 1 . A closed set $\mathcal{P} \in \mathcal{X}$ with the origin in its interior is called controlled $\gamma$-contractive iff, for a given $\gamma \in[0,1)$ and for all $x \in \mathcal{P}$ there exists an $u \in \mathcal{U}$ such that $A x_{k}+B u_{k} \in \gamma \mathcal{P}$.

The contractive set $\mathcal{P}$ for system 1 can be given as $\mathcal{P}=$ $\left\{x \in \mathcal{R}^{n} \mid F x_{k} \leq f\right\}$. It is contractive if $\forall x_{k} \in P \exists u_{k}$ such that

$$
F\left(A x_{k}+B u_{k}\right) \leq \gamma f
$$

In the following an optimization based approach to find a large contractive set will be described. The approach is based on maximizing the volume of an ellipsoid inscribed in the contractive set. Finding the inscribed ellipsoid of maximum volume will be addressed in the next subsection. For the volume of the inscribed ellipsoid to be bounded, the set $\mathrm{P}$ itself needs to be bounded, and additional considerations are necessary to ensure that the set is also controlled contractive with the required contraction factor. These issues will be addressed in subsequent subsections.

\section{A. Maximum Volume Ellipsoid inscribed in a set}

Consider the set $\mathcal{P}$ described by $m$ linear inequalities as $\mathcal{P}=\left\{x \in \mathcal{R}^{n} \mid F_{i} x_{k} \leq f_{i}, \forall i=1 \ldots m\right\}$ and the ellipsoid $\varepsilon$ given as $\varepsilon=\{C y+d \mid\|y\| \leq 1\}$, where $C=C^{T}>0$. According to [17], the volume of ellipsoid $\varepsilon$ is proportional to $\operatorname{det}(C)$. The maximum volume ellipsoid $\varepsilon$ inscribed in $\mathcal{P}$ can be obtained by solving the optimization problem:

$$
\max _{C, d, F, f} \log (\operatorname{det}(C))
$$

subject to

$$
\begin{gathered}
C=C^{T}>0 \\
{\left[\begin{array}{cc}
\left(f_{i}-F_{i} d\right) I & C F_{i}^{T} \\
F_{i} C & f_{i}-F_{i} d
\end{array}\right] \succeq 0 \forall i=1, \ldots m}
\end{gathered}
$$

Alternatively, one may maximize the radius $z$ of a ball $\mathcal{B}$ having the center at $c$ inscribed inside the polytope $\mathcal{P}$.

$$
\mathcal{B}=\left\{x \in \mathcal{R}^{n}\|c-x\|_{2} \leq z\right\}
$$

Where $z$ and $c$ are known as Chebyshev radius and Chebyshev center respectively.

To make sure that the ball is entirely inside $\mathcal{P}$, we ensure that the distance from center $c$ of circle to any hyperplane is greater than or equal to Chebyshev radius $z$ for each constraint $\left\{F_{i} x_{k} \leq f_{i}, \forall i=1, \ldots m\right\}$. Therefore it imposes following constraints:

$$
\left\|F_{i} c-f_{i}\right\| \geq\left\|F_{i}\right\|_{2} z
$$

As $c$ satisfies inequalities $F_{i} c \leq f_{i}$, therefore we have

$$
F_{i} c+\left\|F_{i}\right\| z \leq f_{i}
$$

Thus, by maximizing Chebyshev radius $z$, the largest possible ball within a contractive set can be found.

Using ball $\mathcal{B}$ instead of ellipsoid $\varepsilon$ is simpler but contractive set obtained by inscribing the maximum volume ellipsoid $\varepsilon$, can be much larger than the one obtained by inscribing largest ball $\mathcal{B}$. From here on, we shall consider a maximum volume ellipsoid inscribed in $\mathcal{P}$.

\section{B. Boundedness Constraints}

We find maximum volume ellipsoid $\varepsilon$ inscribed in $\mathcal{P}$ from (3). But this does not guarantee that contractive set obtained by such formulation will be bounded as well. If the volume of the inscribed ellipsoid is finite (the maxdet problem is well posed), the set $\mathcal{P}$ is also bounded. However, we want to impose conditions to make sure that this is the case. To ensure boundedness for contractive set we introduce bounding constraints on set $\mathcal{P}$ so that when $\mathcal{P}$ is bounded by a set $\mathcal{H}$, the inequalities defining $\mathcal{H}$ are redundant when inequalities defining $\mathcal{P}$ are added. $\mathcal{H}$ should contain the state constraints and added lax constraints, if the state constraints set $\mathcal{X}$ is not bounded. Let $\mathcal{H}$ be defined as:

$$
\mathcal{H}=\left\{x \in \mathcal{R}^{n} \mid H x_{k} \leq h\right\}
$$

Then from [13] we know that $\mathcal{P}$ is contained in $\mathcal{H}$ (and hence is bounded), if and only if there exists a matrix $M$ with non-negative elements such that

$$
M F=H
$$

and

$$
M f \leq h
$$

We can find such $M$ which bounds set $\mathcal{P}$ by solving above mentioned problem.

\section{Contractive Constraints}

The set $\mathcal{P}$ is contractive if it fulfills Definition 1 . We want to find $\mathcal{P}=\left\{x \in \mathcal{R}^{n} \mid F x_{k} \leq f\right\}$ with maximum guaranteed contraction factor such that eq. (2) is satisfied. Maximum contraction factor for a given $F$ and $f$ can be found by solving following bi-level optimization problem:

$$
\max _{x} \gamma^{*}
$$

subject to

$$
\begin{gathered}
H x_{k} \leq h \\
F x_{k} \leq f \\
\gamma^{*}=\min _{u, \gamma} \gamma
\end{gathered}
$$

subject to

$$
\begin{gathered}
F\left(A x_{k}+B u_{k}\right) \leq \gamma f \\
H_{u} u \leq h_{u}
\end{gathered}
$$

Here the solution of the lower level problem imposes constraints on upper level problem. By replacing the lower-level 
problem by the corresponding KKT conditions [11], one obtains:

$$
\max _{x, u, \gamma} \gamma^{*}
$$

subject to

$$
\begin{gathered}
H x_{k} \leq h \\
F x_{k} \leq f \\
F\left(A x_{k}+B u_{k}\right)-\gamma f \leq 0 \\
H_{u} u \leq h_{u} \\
\lambda_{a}\left(F\left(A x_{k}+B u_{k}\right)-\gamma f\right) \leq 0 \\
\lambda_{b}\left(H_{u} u-h_{u}\right) \leq 0 \\
\nabla_{u, \gamma} \mathcal{L}(u, \gamma, \lambda) \leq 0
\end{gathered}
$$

where the Lagrangian function $\mathcal{L}$ is given by

$$
\mathcal{L}=\gamma^{*}+\lambda_{a}^{T}\left(F A x+F B u-\gamma h_{x}\right)+\lambda_{b}^{T}\left(H_{u} u-h_{u}\right)
$$

Here $\lambda_{a}$ and $\lambda_{b}$ are Lagrangian multipliers for the inequality constraints. Equations (13f) and (13g) induces nonlinearity in the system. We use binary variables $s \varepsilon\{0,1\}$ to remove this non-linearity as explained in [12] to obtain following single level optimization problem:

$$
\max _{x, u, \gamma, \lambda, s} \gamma^{*}
$$

subject to

$$
\begin{aligned}
& H x_{k} \leq h \\
& F x_{k} \leq f \\
& \lambda_{a} \geq 0 \\
& \lambda_{b} \geq 0 \\
& \lambda_{a} \leq M_{a}^{\lambda} s \\
& \lambda_{b} \leq M_{b}^{\lambda} s \\
& F\left(A x_{k}+B u_{k}\right)-\gamma f \leq 0 \\
& \lambda\left(F\left(A x_{k}+B u_{k}\right)-\gamma f\right) \geq-M_{a}^{u}(1-s) \\
& H_{u} u \leq h_{u} \\
& H_{u} u-h_{u} \geq-M_{b}^{u}(1-s) \\
& \nabla_{u, \gamma} \mathcal{L}(u, \gamma)=0
\end{aligned}
$$

Here $M_{a}^{\lambda}, M_{b}^{\lambda}, M_{a}^{u}$ and $M_{b}^{u}$ are diagonal matrices with appropriate dimensions and sufficiently large elements on the main diagonal. If a candidate contractive set is given, its maximum contraction factor can be found using the formulation described above.

\section{Problem Formulation}

Previous subsections imposed the constraints on $\mathcal{P}$. We want to maximize volume of an ellipsoid $\varepsilon$ so that it is inscribed in contractive set $\mathcal{P}$ such that boundedness and contractive constraints are satisfied. Thus we have a following optimization problem to obtain $\mathcal{P}$ :

$$
\max _{C, d, F, f} \log (\operatorname{det}(C))
$$

subject to

$$
\begin{gathered}
C=C^{T}>0 \\
{\left[\begin{array}{cc}
\left(f_{i}-F_{i} d\right) I & C F_{i}^{T} \\
F_{i} C & f_{i}-F_{i} d
\end{array}\right] \succeq 0 \forall i=1, \ldots m} \\
M F=H \\
M f \leq h \\
\max _{x, u, \gamma, \lambda, s} \gamma^{*}
\end{gathered}
$$

subject to

$$
\begin{aligned}
& H x_{k} \leq h \\
& F x_{k} \leq f \\
& \lambda_{a} \geq 0 \\
& \lambda_{b} \geq 0 \\
& \lambda_{a} \leq M_{a}^{\lambda} s \\
& \lambda_{b} \leq M_{b}^{\lambda} s \\
& F\left(A x_{k}+B u_{k}\right)-\gamma f \leq 0 \\
& \lambda\left(F\left(A x_{k}+B u_{k}\right)-\gamma f\right) \geq-M_{a}^{u}(1-s) \\
& H_{u} u \leq h_{u} \\
& H_{u} u-h_{u} \geq-M_{b}^{u}(1-s) \\
& \nabla_{u, \gamma} \mathcal{L}(u, \gamma)=0
\end{aligned}
$$

The problem formulated above can be used to find the maximum volume ellipsoid $\varepsilon$ and the corresponding contractive set $\mathcal{P}=\left\{x \in \mathcal{R}^{n} \mid F x_{k} \leq f\right\}$ inside which the ellipsoid $\varepsilon$ resides. The advantage of this method is that the complexity of contractive set is flexible. There is a tradeoff between complexity of the contractive set reduced and volume of the set. The complexity of the contractive set can be selected according to the specific application. Note that problem formulated above can be simplified by removing parameter $f$ by normalizing set $\mathcal{P}$ such that $\mathcal{P}=\{x \in$ $\left.\mathcal{R}^{n} \mid F x_{k} \leq \mathbb{1}\right\}$. If the problem formulation is symmetric, this can be exploited to further simplify the computations. Example VI-A shows the effectiveness of this algorithm by comparing the results with the method described in [10]. The contractive sets obtained of different complexities for the system with identical modes are shown in example VIB. 


\section{E. Solving the Formulated Problem}

For the optimization problem in eq (16), the possible simplifications mentioned in the previous subsection notwithstanding, is a large optimization problem with highly nonconvex constraints. Solving this problem to a (provable) global optimality is therefore very difficult and computationally costly. Luckily, for this problem a solution need not be globally optimal in order to be useful. In this work, Particle Swarm Optimization (PSO) is used to find good solutions to the problem in eq (16).

PSO technique described in [15] is used to solve problem formulated in previous subsection. It is a population based stochastic method. Particles, candidate solution, are moved around search space with certain position and velocity. Each particle is moved towards best position in search space, so we move the particle swarm towards best solutions. Particle Swarm Optimization is an unconstrained optimization. In order to apply PSO, eq (16) is converted into an unconstrained problem by appending suitable penalty functions accounting for constraint violations to the optimization criterion.

While exploring the search space, the PSO typically finds multiple feasible solutions. Such solutions can be merged to obtain an enlarged contractive set, as explained in the next section.

\section{Merging of Contractive Sets}

Definition 2: The convex hull of a family of sets $S$ is the smallest convex set containing the entire family of sets $S$.

The volume of the contractive set can be increased by finding the convex hull of different solutions obtained by the Particle Swarm Optimization. Unfortunately, it will not only increase the volume of the contractive set but will also increase the complexity of set.

Theorem 1: The convex hull of the contractive sets $S_{1}$ and $S_{2}$ with contraction factors $\gamma_{1}$ and $\gamma_{2}$ respectively, is $\gamma$ contractive, where $\gamma=\max \left(\gamma_{1}, \gamma_{2}\right)$.

Proof: Let $x_{1}(k)$ and $x_{2}(k)$ be points on the contractive sets $P_{1}$ and $P_{2}$ respectively whose convex hull is denoted by $P_{0}$. Assume that contraction factors for $P_{1}$ and $P_{2}$ are $\gamma_{1}$ and $\gamma_{2}$ respectively. Then for $x_{1}(k) \exists u_{1}(k) \in U \mid x_{1}(k+1) \in \gamma_{1} P_{1}$ and $x_{2}(k) \exists u_{2} \in U \mid x_{2}(k+1) \in \gamma_{2} P_{2}$. Let $x_{0}(k)$ be point obtained outside $P_{1}$ and $P_{2}$ but inside the convex hull. Then, $x_{0}(k)$ can be expressed as:

$$
x_{0}(k)=\alpha_{1} x_{1}(k)+\alpha_{2} x_{2}(k)
$$

where $\alpha_{1}, \alpha_{2} \geq 0, \alpha_{1}+\alpha_{2} \leq 1$ Convex hull is contractive if and only if there exist $\left\{u_{0}(k) \in U \mid x_{0}(k+1) \in \gamma P_{0}\right\}$. Applying the input,

$$
u_{0}(k)=\alpha_{1} u_{1}(k)+\alpha_{2} u_{2}(k)
$$

the system dynamics (1) results in

$$
\begin{array}{r}
x_{0}(k+1)=A\left(\alpha_{1} x_{1}(k)+\alpha_{2} x_{2}(k)\right)+B\left(\alpha_{1} u_{1}(k)+\alpha_{2} u_{2}(k)\right) \\
x_{0}(k+1)=\alpha_{1}\left(A x_{1}(k)+B u_{1}(k)\right)+\alpha_{2}\left(A x_{2}(k)+B u_{2}(k)\right)
\end{array}
$$

$$
x_{0}(k+1)=\alpha_{1} x_{1}(k+1)+\alpha_{2} x_{2}(k+1)
$$

As $x_{1}(k+1) \in \gamma_{1} P_{1}$ and $x_{2}(k+1) \in \gamma_{2} P_{2}$, therefore

$$
x_{0}(k+1) \in \gamma\left(\alpha_{1} P_{1} \oplus \alpha_{2} P_{2}\right) \in \gamma P_{0}
$$

\section{Simplification of Contractive Sets}

Assume that the contractive set obtained after taking the convex hull of different solutions provided by the PSO is $\mathcal{P}_{c}$ with $m$ hyperplanes and $n v$ number of vertices. We want to minimize the number of hyperplanes so that the complexity of the formulated contractive set is reduced. As such a convex hull for higher dimensional systems can be complex, we'd like to reduce its complexity. Naturally, this complexity reduction should be obtained without reducing the volume of the contractive set substantially, and without increasing the contraction factor much. We introduce two methods to obtain the simplified contractive sets. The first method obtains the new set of vertices by using a circumscribed ellipsoid while the second method removes the hyperplanes so that the set $\mathcal{P}_{c}$ remains contractive with required contraction factor within state constraints.

\section{A. Circumscribed Ellipsoid}

In this method we find a minimum volume ellipsoid $\varepsilon_{c}=\{\|C x+d\| \leq 1\}$ with center at $d$ circumscribing the contractive set $\mathcal{P}_{c}$. The ellipsoid $\varepsilon_{c}$ can be found by minimizing the volume of ellipsoid containing all vertices of $\mathcal{P}_{c}$, this results in a maxdet problem as follows:

$$
\min _{C, d}-\log (\operatorname{det}(C))
$$

subject to

$$
\begin{gathered}
C=C^{T}>0 \\
\left\|C V_{i}+d\right\| \leq 1
\end{gathered}
$$

Where $V_{i}$ is a vertex of $\mathcal{P}_{c}$, and $n v$ is the number of vertices of $\mathcal{P}_{c}$. Once the circumscribed ellipsoid is found, we look at which points from $V_{i}$ lie on the ellipsoidal boundary. Let us assume vertices $V_{E}$ lie on the boundary of the ellipsoid such that $\left\{V_{E} \in V_{i}, \forall i=1, . . n v\right\}$. Then we rotate the ellipsoid such that point $p_{f}$ in $V_{i}$, which was farthest from ellipsoidal boundary lies on new rotated ellipsoid. This adds the vertex which will give the largest increment in the volume. We find a normal plane to vector passing through $p_{f}$ and center of ellipsoid. There can be many directions in which such a plane can lie. We take projection $\varepsilon_{p}$ of the ellipsoid $\varepsilon_{c}$ and points $V_{i}$ on the plane and then check which new point is farthest from $\varepsilon_{p}$. We add that point to current point $p_{f}$ and continue with projections. This procedure is repeated for $n-1$ times to obtain $n-1$ points. Normal to these $n-1$ points will give us a specific direction for rotation of ellipsoid (orientation of plane). We add two points on the new plane far away from original points and find a new minimum volume ellipsoid inscribing all the points. It makes sure that the point $p_{f}$ lies on boundary of the new ellipsoid. In this way, the ellipsoid will be rotated to find new set of vertices which originally belongs to $V_{i}$, common points of the ellipsoid and the contractive set are 
added to $V_{E}$. We repeat the rotation procedure until we find a large enough contractive set. $V_{E}$ forms the vertices of new simplified contractive set. There is a trade-off between volume of contractive set obtained and complexity reduced.

\section{B. Removing a Hyperplane}

The method described above finds a reduced complexity contractive set. As the method focuses on vertex operation for complexity reduction, there may arise a case when it increases the complexity in terms of hyperplanes, in that case the method described above should be discarded. Here, we propose a method for simplification of a polytope by directly operating on the hyperplanes. We remove the hyperplane if contraction factor of the simplified set doesn't exceed the required contraction constraint. We select the hyperplane $H_{i}$ to be removed by checking which hyperplane has to be pushed outwards least, in order to become redundant. The hyperplane $H_{i}$ can be removed if new vertices $V$ obtained by removing $H_{i}$ are contractive and also fulfills the state constraints. The procedure is repeated for all the hyperplanes. As this method directly removes the hyperplanes, it guarantees complexity reduction and increase in volume of the contractive set. In worst case scenario, the procedure will not have any impact on the set unlike method described in previous sub-section. Results obtained with this procedure along with the formulations of previous method are illustrated in example VI-A.

\section{Designing a Controller With Given CONTRACTIVE SET}

Assume the low complexity controlled contractive set with contraction factor $\gamma$ is obtained by the methods mentioned above. Then, the control formulation can be given as:

$$
\min _{u_{k}} x_{k+1}^{T} Q x_{k+1}+u_{k}^{T} R u_{k}
$$

subject to

$$
\begin{gathered}
x_{k+1}=A x_{k}+B u_{k} \\
H_{u} u \leq h_{u} \\
F x_{k+1} \leq \gamma \alpha f
\end{gathered}
$$

where

$$
\alpha=\max \left\{F_{i} x_{k, i} / f_{i}\right\}, \forall i=1, \ldots, m
$$

The contractiveness ensures that the $\alpha$ will reduce by factor $\gamma$ at each time step, therefore the state trajectories will converge to origin, which ensures stability of the system. The explicit solution to (22) can be obtained by solving it parametrically, with $x_{k}$ and $\alpha$ as the parameters. The complexity of the explicit solution (in terms of the number of critical regions obtained), is given by the number of different combinations of constraints that may be active at the optimum, when the parameters are allowed to vary throughout the given parameter region. MPC formulations with a long prediction horizon will have a high number of constraints, and thus also typically a high number of possible combinations of different constraints. The prediction horizon for the formulation in (22) is 1 , and thus the complexity of the explicit solution can be expected to be low.

\section{EXAMPLES}

\section{A. Spring Mass Damper system}

Consider the spring mass damper system example mentioned in [4] with state representation given as:

$$
\dot{x}=\left[\begin{array}{cc}
0 & 1 \\
-7 & -7
\end{array}\right] x+\left[\begin{array}{l}
0 \\
1
\end{array}\right] u
$$

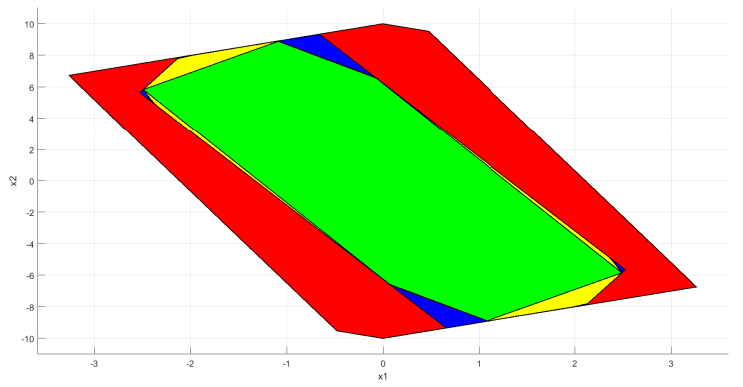

Fig. 1: Complexity Reduction of Contractive Set

System is discretized with sampling time of $0.01 \mathrm{sec}$, input and state constraints are given as $-10 \leq u \leq 10$ and $-10 \leq x_{i} \leq 10, \forall i=1,2$ respectively. First we find a contractive set by method proposed in section II. It comes out to be a set with contraction factor $\gamma=0.9796$, volume of 32.7378 and 6 hyperplanes. Set in red obtained after 200 iterations of Dorea-Hennet procedure, has volume 71.7814 with 91 hyperplanes (Red set in figure 1). The contractive set obtained by the method described in section III has 20 hyperplanes with volume of 40.9193 and contraction factor 0.9774 (Yellow set in figure 1). By simplifying the larger contractive set using method explained in section IVA, the set obtained has a volume of 38.2663 with 0.9771 contraction factor and 6 complexity (as shown in green in figure 1). By implementing method described in section IV$\mathrm{B}$, simplified contractive set obtained has 6 hyperplanes with volume 42.4433 and contraction factor 0.9783 (blue set).

\section{B. Identical Modes system}

Consider a system with identical modes in series, i.e, nondiagonalizable system matrix A given as:

$$
x_{k+1}=\left[\begin{array}{cc}
0.98 & 1 \\
0 & 0.98
\end{array}\right] x+\left[\begin{array}{cc}
0 & 0 \\
0.6 & 0.9
\end{array}\right] u
$$

The contractive sets obtained for this system are shown in figure 2. Contractive set obtained by the method explained in [10] is shown in blue, while the green, red and yellow sets are obtained using the optimization based approach in this paper, when specifying different set complexities. It can be clearly seen that by decreasing complexity of a set, we also lose volume. Therefore, there is a trade-off between the volume of the contractive set and its complexity. 


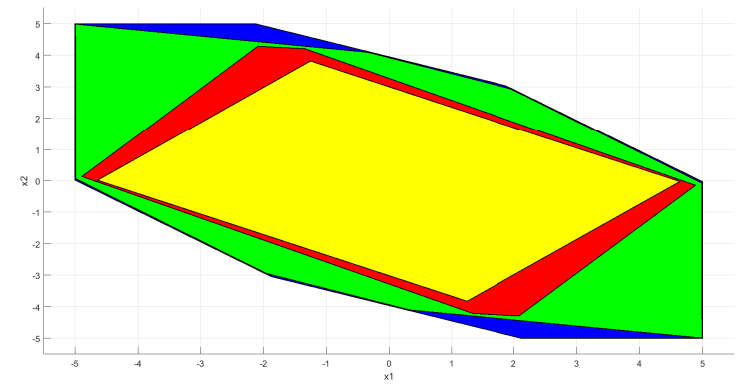

Fig. 2: Flexible Complexity Contractive Sets

\section{Higher dimensional system with the controller design}

Consider the following system:

$x_{k+1}=\left[\begin{array}{ccc}0.98 & 0 & 0 \\ 0.53 & 0.98 & 0 \\ -0.65 & 0.52 & 0.98\end{array}\right] x_{k}+\left[\begin{array}{cc}0.1 & 0 \\ 0.2 & 0.1 \\ 0 & 0.3\end{array}\right]_{(25)}$

Input and state constraints are given as $-1 \leq u_{i} \leq$ $1, \forall i=1,2$ and $-1 \leq x_{j} \leq 1, \forall j=1, \ldots 3$ respectively. The contraction factor is chosen to be 0.9745 . The explicit solution for a MPC with prediction horizon of 10 has 862 regions, and the total volume of the feasible region is 6.77 . The contractive set for the system as explained in sections II is obtained with complexity of 8 and volume 2.0828 . The controller is designed by method explained in section V. By using the set obtained from section II formulations, number of regions comes out to be 21 . Clearly, the reduction in volume is significant, but so is the reduction in complexity for the explicit controller.

\section{CONCLUSION}

A novel method for formulating controlled contractive sets has been described in this paper. An optimization based approach is used to find the contractive sets, subject to boundedness and contractiveness constraints. Multiple contractive sets can subsequently be merged to obtain a larger contractive set. Two techniques have been discussed to further simplify the set. The first technique utilizes circumscribed ellipsoid to find a reduced complexity contractive set while second technique proposes an operation on the hyperplanes to reduce the complexity. Finally, numerical examples shows the efficiency of the proposed method.

\section{REFERENCES}

[1] Morimasa Ogawa and Manabu Kano Practice and Challenges in Chemical Process Control Applications in Japan, Proceedings of the 17th World Congress The International Federation of Automatic Control Seoul, Korea, July 6-11, 2008

[2] D.Hrovat, S. Di Cairano, H.E. Tseng andI.V. Kolmanovsky The Development of Model Predictive Control in Automotive Industry: A Survey, 2012 IEEE International Conference on Control Applications (CCA) Part of 2012 IEEE Multi-Conference on Systems and Control October 3-5, 2012. Dubrovnik, Croatia

[3] K. S. Holkar and L. M. Waghmare An Overview of Model Predictive Control, International Journal of Control and Automation Vol. 3 No. 4 , December, 2010
[4] Morten Hovd, Sorin Olaru and George Bitsoris Low Complexity Constraint Control Using Contractive Sets. Preprints of the 19th World Congress, The International Federation of Automatic Control, Cape Town, South Africa. August 24-29, 2014

[5] Francesco Scibilia Explicit Model Predictive Control: Solutions Via Computational Geometry, Thesis for the degree of philosophiae doctor, November 2010 -NTNU, Norway

[6] Alberto Bemporad, Manfred Morari, Vivek Dua, Efstratios N. Pistikopoulos The explicit linear quadratic regulator for constrained systems, Automatica Volume 38, Issue 1, January 2002, Pages 3-20

[7] C. N. Jones and M. Morari Approximate explicit mpc using bilevel optimization. Proceedings of the European Control Conference 2009, Budapest, Hungary, August 23-26, 2009

[8] Hoai-Nam Nguyen Implicit improved vertex control for uncertain, timevarying linear discrete-time systems with state and control constraints. Automatica, 49(9):2754- 2759, 2013.

[9] F. Scibilia, S. Olaru, M. Hovd On feasible sets for MPC and their approximations. Automatica, 47(1) - pp.133-139, 2011.

[10] C. Dorea and J. C. Hennet. $(a, b)$-invariant polyhedral sets for linear discrete-time systems. Journal of Optimization Theory and Applications, 103(3):521-542, 1999.

[11] Morten Hovd. Multi-level Programming for Designing Penalty Functions for MPC Controllers. Preprints of the 18th IFAC World Congress Milano (Italy) August 28 - September 2, 2011

[12] J. Fortuny-Amat and B. McCarl A representation and economic interpretation of a two-level programming problem. Journal of the Operational Research Society,32(9), 783-792, Sep 1981.

[13] G. Bitsoris On the Positive Invaraince of polyhedral sets for discrete -time systems. System and Control letters 1988, 11(3), 243-248.

[14] Jialin Han, Guangquan Zhang, Yaoguang Hu, Jie Lu Solving Trilevel Programming Problems Using a Particle Swarm Optimization Algorithm. 2015 IEEE 10th Conference on Industrial Electronics and Applications (ICIEA)

[15] F. Van den Bergh, A. P.E ngelbrecht A New Locally Convergent Particle Swarm Optimiser. 2002 IEEE International Conference on Systems, Man and Cybernetics, Volume 3, 6-9 Oct. 2002

[16] Tobias Geyer ,Fabio D. Torrisi, Manfred Morari Optimal complexity reduction of polyhedral piecewise affine systems. Automatica 44 (2008) $1728-1740$

[17] Lieven Vandenberghe ,Stephen Boyd, Shao-Po Wu Determinant maximization with linear matrix inequality constraints. SIAM Journal on Matrix Analysis and Applications, 19(2):499-533, 1998. 